\title{
EPIDOR: uma abordagem computacional baseada em sistema web e aplicativo móvel para dores crônicas no atual contexto de pandemia do Coronavírus
}

\section{EPIDOR: computational environment based on web system and app for chronic pain in the current Coronavirus pandemic context}

\author{
Fabrício Martins Mendonça ${ }^{1}$, Carlos Alberto Mourão Junior ${ }^{2}$, Juan Felipe Souza Oliveira ${ }^{3}$, Thiago Goldoni \\ Thomé ${ }^{4}$, Marcelo Quesado Filgueiras ${ }^{5}$ \\ 1 Universidade Federal de Juiz de Fora (UFJF), Juiz de Fora, Minas Gerais, Brasil. ORCID: 0000-0001-8156-8607 \\ 2 Universidade Federal de Juiz de Fora (UFJF), Juiz de Fora, Minas Gerais, Brasil. ORCID: 0000-0001-7199-5365 \\ ${ }^{3}$ Universidade Federal de Juiz de Fora (UFJF), Juiz de Fora, Minas Gerais, Brasil. ORCID: 0000-0002-8380-4017 \\ ${ }^{4}$ Universidade Federal de Juiz de Fora (UFJF), Juiz de Fora, Minas Gerais, Brasil. ORCID: 0000-0002-4984-6379 \\ ${ }^{5}$ Universidade Federal de Juiz de Fora (UFJF), Juiz de Fora, Minas Gerais, Brasil. ORCID: 0000-0002-3862-7197
}

Autor para correspondência/Mail to: Fabrício Martins Mendonça, fabriciommendonca@gmail.com

Recebido/Submitted: 26 de junho de 2020; Aceito/Approved: 04 de setembro de 2020

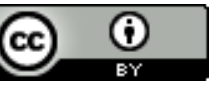

Copyright (c) 2020 Mendonça, Mourão Junior, Oliveira, Thomé \& Filgueiras. Todo o conteúdo da Revista (incluindo-se instruções, política editorial e modelos) está
sob uma licença Creative Commons Atribuição 4.0 Internacional. Ao serem publicados por esta Revista, os artigos são de livre uso em ambientes educacionais,
de pesquisa e não comerciais, com atribuição de autoria obrigatória. Mais informações em http://revistas.ufpr.br/atoz/about/submissions\#copyrightNotice.

\begin{abstract}
Resumo
Introdução: Investiga os problemas relacionados ao contexto informacional no cenário de pandemia, especificamente, aqueles referentes a pacientes portadores de dor crônica, os quais representam um dos grupos vulneráveis à COVID-19. Apresenta como solução uma abordagem computacional baseada em um sistema web e aplicativo móvel para dores crônicas, denominado EPIDOR, capaz de contribuir com o processo de transformação digital na área. Método: O EPIDOR foi desenvolvido com base no levantamento de requisitos sobre dor crônica na literatura da área e com especialistas médicos, os quais também fizeram a validação dos requisitos do sistema desenvolvido. É caracterizada como pesquisa aplicada, exploratória e bibliográfica. Conduz um estudo de caso em um hospital da rede pública e uma clínica especializada em medicina da dor com uma amostra de pacientes portadores de dor crônica, os quais terão registrados sua intensidade da dor diária ao longo de um período, sua correlação com atividades e horário de medicação. Resultados: O sistema EPIDOR faz parte de uma pesquisa em andamento e se encontra em fase de implantação no hospital e clínica especializada mencionados. Fornece suporte para estudos epidemiológicos da dor no Brasil. Estes ainda carecem de dados mais confiáveis e robustos acerca da incidência e prevalência da dor em âmbito nacional e regional, especialmente no contexto atual de pandemia da COVID-19. Conclusão: Conclui que o sistema EPIDOR pode contribuir no processo de transformação digital na área de dor crônica. Também pode prover uma melhoria da qualidade de vida desse grupo de pacientes. Enfatiza-se que esse grupo corresponde a mais de $30 \%$ da população brasileira
\end{abstract}

Palavras-chave: Dor crônica; COVID-19; Coronavírus; Transformação Digital; Sistema de Informação Eletrônico.

\begin{abstract}
Introduction: It investigates the problems related to the informational context in the pandemic scenario, specifically those related to patients with chronic pain, which represent one of the groups vulnerable to COVID-19. This article presents a computational approach based on a web system and mobile application solution for chronic pain, called EPIDOR, capable of contributing to the process of digital transformation in the area. Method: The EPIDOR was developed based on the survey of requirements on chronic pain in the literature of the area, and with medical specialists, who also validated the requirements of the developed system. It is applied, exploratory and bibliographic research. It conducts a case study in a public hospital and a clinic specializing in pain medicine with a sample of patients with chronic pain, who will have recorded their daily pain intensity over a period, its correlation with activities and medication schedule. Results: The EPIDOR system is part of ongoing research and is being implemented in a public hospital and a clinic specialized in pain medicine. It provides support for epidemiological studies of pain in Brazil. These still lack more reliable and robust data on the incidence and prevalence of pain at the national and regional levels, especially in the current context of the COVID-19 pandemic. Conclusion: It concludes that the EPIDOR system can contribute to the digital transformation process in the area of chronic pain. The proposed approach can provide an improvement in the quality of life of this group of patients. We emphasize that this group corresponds to more than $30 \%$ of the Brazilian population.

Keywords: Chronic Pain; COVID-19; Coronavirus; Digital Transformation; Electronic Information System.
\end{abstract}

\section{INTRODUÇÃO}

Os serviços de saúde estão enfrentando, cada vez mais, o desafio de acessibilidade para uma população em crescimento e envelhecimento, além do surgimento de epidemias globais, como a atual pandemia do novo coronavírus (SARS-CoV-2). Além das fragilidades na infraestrutura de saúde, a pandemia atual expôs o problema da falta de informação precisa para processos de tomada de decisão nos níveis de gestão pública, logístico e operacional e também para apoiar profissionais da área de saúde em decisões clínicas e diagnóstico. Em outras palavras, é possível inferir que a transformação digital, processo de mudança realizado a partir do uso das chamadas tecnologias sociais, móveis, analíticas e tecnologias de computação em nuvem capazes de 
gerar inovações nas dimensões individual, social e empresarial (Toolboom, 2016), não ocorre como seria esperado em todo mundo, especialmente no contexto da saúde. Especificamente na área de saúde, faz-se necessário um melhor entendimento do uso dessas novas tecnologias, políticas públicas de incentivo à sua utilização, leis regulamentadoras da privacidade de dados e uso ético da informação. O presente artigo defende a ideia da adoção prática e uso eficiente de tecnologias para transformação digital como uma solução apropriada para enfrentar o desafio cada vez maior de acessibilidade aos serviços de saúde pela população, ampliado em cenários de pandemia.

Uma área da saúde que deveria merecer destaque normalmente e agora ainda mais com a pandemia do coronavírus é a medicina da dor. A dor crônica é uma questão de saúde pública com altos índices de morbidade, absenteísmo ao trabalho e incapacitação temporária ou permanente com custos elevados para as fontes financiadoras da saúde (Sá, Baptista, Matos, \& Lessa, 2009). Estima-se que a prevalência de dor crônica no mundo esteja em torno de $7 \%$ a 40\% da população mundial sofre de dor crônica (Dellaroza, Pimenta, e Matsuo, 2007; Fishbain et al., 2007). No Brasil, embora não haja muitos estudos epidemiológicos, algumas pesquisas confirmam incidência semelhante a essa estimada pela International Association for the Study of Pain (IASP) com uma média de prevalência em torno de 35,5\% (Vasconcelos \& Araújo, 2018) com grandes variações nos diferentes estados brasileiros.

A dor crônica, apesar de estar aumentando no mundo, continua a ser subestimada tanto como problema de saúde quanto como fator comprometedor da qualidade de vida da população. O impacto financeiro da dor crônica nos sistemas de saúde, público e privado, ainda é pouco conhecido e o Brasil parece estar ainda longe de ter dados epidemiológicos confiáveis e robustos acerca da incidência e prevalência da dor em âmbito nacional e regional (Carvalho et al., 2018). Estudos mais aprofundados sobre a epidemiologia da dor e a geração de dados eletrônicos mais abrangentes e confiáveis se fazem necessários (Andrade, 2014). É dentro deste contexto que se insere a presente pesquisa.

Além dos problemas já conhecidos sobre epidemiologia da dor, o cenário atual de pandemia do coronavírus introduz novos problemas que impactam diretamente no tratamento da dor crônica, estabelecendo possíveis relações entre a COVID-19 e dores crônicas. Na presente pesquisa, os problemas levantados como complicadores à dor crônica foram os seguintes: i) afastamento de pacientes com dor crônica de seu tratamento (presencial ou à distância) com medo de contrair a COVID-19 ou mesmo desconhecimento do acompanhamento médico remoto; ii) agravos de problemas da população idosa, que naturalmente são maiores portadores de dor crônica; e iii) problemas de saúde mental gerados pela pandemia e por conta das atuais fake news, com enfoque na relação entre ansiedade e dor crônica.

Diante deste contexto, o objetivo da presente pesquisa é apresentar um ambiente computacional para coleta, acompanhamento e gestão de dados de pacientes com dores crônicas, denominado EPIDOR (acrônimo de Epidemiologia da Dor), que possa contribuir com estudos epidemiológicos mais completos e detalhados sobre dor crônica no Brasil. O sistema é composto por dois componentes de software (um sistema web e um aplicativo móvel), que possibilitam a coleta de dados de dores crônicas, registro eletrônico da intensidade da dor ao longo de um período, sua correlação com atividades e horário de medicação, além da geração de dashboards dos dados obtidos a fim de prover análise de dados para tomada de decisão gerencial.

Para cumprir com seu propósito, o presente artigo foi estruturado nas seguintes seções: a primeira seção trouxe a introdução do tema com contexto, justificativa e objetivos. A Seção 2 descreve a fundamentação teórica desta pesquisa a partir de dois assuntos principais: possíveis relações entre dor crônica e a COVID-19 no atual cenário de pandemia (subseção 2.1) e o papel da transformação digital diante de tal cenário (subseção 2.2). A Seção 3 apresenta a metodologia de pesquisa (subseção 3.1) e o ambiente computacional EPIDOR com seus dois componentes de software: o sistema web (subseção 3.2) e o aplicativo móvel (subseção 3.3). A Seção 4 apresenta uma discussão sobre a proposta desta pesquisa e seus resultados esperados. Por fim, a Seção 5 traz considerações finais da pesquisa.

\section{FUNDAMENTAÇÃO TEÓRICA}

\section{COVID-19 e Dor Crônica}

Na literatura médica, dor crônica é definida como aquela que tem duração maior que três meses depois da injúria que a causou, ou seis meses para fins de pesquisa, sendo que estudos recentes sobre o tema mostram que grande parte do que foi coletado de informações no Brasil remontam aos últimos 10 anos, com prevalência compreendida de 29,3 a 73,3\%, sendo maior em mulheres (Vasconcelos \& Araújo, 2018). É importante destacar também que, em nível nacional, temos poucos estudos sobre epidemiologia da dor e a maior parte deles foi conduzida em situações específicas e seletivas, tais como em população de idosos (Dellaroza et al., 2007), população adulta (Kreling, Cruz, \& Pimenta, 2006) e grupos de fumantes (Fishbain et al., 2007), além de estudos de uma determinada região do corpo e não considerando o fenômeno da dor crônica como um todo, por exemplo, estudo de dores de cabeça (Queiroz, Barea, \& Blank, 2006) e dores muscular-esquelética (Teixeira et al., 2001). 
Além dos problemas já conhecidos em epidemiologia da dor, o cenário atual de pandemia do novo coronavírus acrescenta fatores complicadores no tratamento de pacientes com dor crônica. Nesse sentido, esta subseção do artigo aponta alguns problemas causados pela pandemia que impactam diretamente no tratamento da dor crônica, estabelecendo possíveis relações entre a COVID-19 e dores crônicas. Especificamente, abordam-se problemas relacionados com o afastamento dos pacientes com dor crônica de seu tratamento (presencial ou à distância), agravos de problemas da população idosa e problemas de saúde mental gerados pela pandemia e por conta das atuais fake news. Tais assuntos não são esgotados no presente artigo, dada a amplitude dos temas, que servem de background teórico para esta pesquisa.

No atual contexto de pandemia do novo coronavírus, vem ocorrendo um fenômeno comum a todos pacientes que necessitam de acompanhamento médico neste cenário: o medo do risco de contágio tem levado os pacientes a deixar de procurar atendimento médico - algo preocupante considerando a gravidade de muitos pacientes com dor crônica. Wellington Briques (médico especialista da Spectrum Therapeutics) afirma que "a epidemia faz com que muitos diagnósticos se atrasem, porque as pessoas têm medo de buscar ajuda médica. Por isso, é importante reforçar o conhecimento das doenças com dor crônica, como é o caso da fibromialgia" (Rede Diário de Comunicação, 2020). O médico acrescenta ainda que os índices atuais de prevalência da dor crônica são alarmantes e preocupantes, especialmente em uma época de pandemia, em que os serviços de saúde estão sofrendo diversos tipos de pressão. Nesse contexto, é preciso buscar novos tratamentos, com mais benefícios e menos efeitos colaterais.

Outro fato que corrobora com a diminuição da assistência médica regular em tempos de epidemia é o afastamento de profissionais de saúde, que não lidam diretamente com a doença contagiosa, de suas atividades presenciais. Em pesquisa recente, Minghelli et al. (2020) fizeram um levantamento do número de fisioterapeutas que interromperam suas atividades presenciais em Portugal para evitar maior risco de exposição ao coronavírus. Dos 619 fisioterapeutas consultados, $73.2 \%$ interromperam suas atividades presenciais e $26.8 \%$ continuaram a trabalhar presencialmente. Dos 453 fisioterapeutas que interromperam o atendimento presencial, $58.9 \%$ continuam a acompanhar os seus pacientes a distância e 41.1\% não têm acompanhado. Embora esses dados não possam ser generalizados para os serviços de fisioterapia, eles levantam um aspecto interessante em tempos de epidemia: diminuição do atendimento fisioterápico a pacientes com tal necessidade, como é o caso dos portadores de dores crônicas. Não se advoga aqui que fisioterapeutas ou outros profissionais da saúde tenham que trabalhar presencialmente expondo-se ao maior risco de contaminação, mas sim incentivar o uso de tecnologias para acompanhamento dos pacientes a distância, como apontado em Minghelli et al. (2020), que destaca o uso de prescrição eletrônica, realização de vídeos explicativos e tratamento por videoconferência de forma síncrona como medidas apropriadas de monitoramento a distância.

O afastamento do acompanhamento médico acomete toda a população em geral que sofre de dor crônica, mas seus males podem ser mais prejudiciais entre os idosos, os quais já sofrem mais naturalmente com dores crônicas. Em razão disso, a Organização Pan-Americana da Saúde [OPS/OMS]. Unidade de Saúde Mental e Uso de Substâncias( La unidad de Salud Mental y Uso de Sustancias, Organização Pan-Americana da Saúde [OPS/OMS]) chama a atenção das autoridades governamentais para os impactos da COVID-19 em sua população, especialmente em indivíduos que se encontram em condição de exclusão social, idosos e com doenças crônicas (OPS/OMS, 2016).

Estudos chineses recentes apontam para essa necessidade do cuidado à saúde de idosos em tempo de pandemia. Yang et al. (2020), por exemplo, ressaltam a necessidade de intervenções personalizadas no cuidado à saúde de idosos nesse contexto. Williams (2020) afirma que a grande população chinesa, envelhecida e com maior suscetibilidade ao COVID-19, tem sofrido mais com suas condições psiquiátricas e passado por maior sentimento de angústia. Além disso, os idosos podem não ter acesso aos cuidados de saúde como resultado das restrições de quarentena em massa e fechamento do transporte público (Yang et al., 2020).

A saúde mental e as condições psicológicas da população no contexto de pandemia são outros aspectos de grande importância que devem ser discutidos e que influenciam também pacientes com dores crônicas. Nesse sentido, Lima et al. (2020) destacam que o rápido avanço da doença e o excesso de informações disponíveis, algumas vezes discordantes, se tornam um ambiente favorável para alterações comportamentais impulsionadoras de adoecimento psicológico, que podem gerar consequências graves na saúde mental do indivíduo. As várias notícias negativas exibidas pela mídia sobre a COVID-19, além das fake news, podem gerar nos indivíduos o estado de alerta constante, correlacionado ao medo de se contaminar e de morrer.

Nessa perspectiva, pode-se afirmar que, juntamente com a pandemia de COVID-19, surge um estado de pânico social em nível global e a sensação do isolamento social desencadeia sentimentos, como angústia, insegurança e medo, que podem se estender até mesmo após o controle do vírus (Hossain, Sultana, \& Purohit, 2020). Em pesquisa recente, Pereira et al. (2020) observaram que os sintomas psicológicos mais comuns identificados nos indivíduos foram estresse, medo, pânico, ansiedade, culpa e tristeza, os quais geram sofrimento psíquico e podem ocasionar o surgimento de transtornos de pânico, transtornos de ansiedade, Transtorno de Estresse Pós-Traumático (TEPT) e depressão. 
Os sentimentos psicológicos de angústia e ansiedade têm um impacto negativo direto em pacientes com dores crônicas, porque pessoas mais ansiosas e portadores de doenças crônicas já carregam uma dose de ansiedade superior ao normal. A ansiedade, assim como a dor, é um mecanismo de alarme. Ela avisa sobre algo perigoso e motiva fazer algo a respeito. Disparos constantes de cortisol progressivamente vão minando as defesas do organismo e prejudicando o desempenho do sistema psico-neuro-imuno-endocrinólogo, que agrupa o cérebro, o sistema nervoso, os órgãos e as células imunes e glândulas endócrinas que, interconectadas, determinam o equilíbrio fisiológico e comportamental (Haddad, Saadé, \& Safieh-Garabedian, 2002). Em resumo, o paciente crônico ansioso sofre mais riscos que o paciente crônico mentalmente equilibrado.

Ademais, é importante ressaltar que a dor crônica é a situação que tem maior potencial de produzir estresse e hipersecreção de adrenalina e cortisol (Vachon-Presseau et al., 2013), e já está bem estabelecido na literatura que o estresse crônico tem forte efeito imunossupressor, ou seja, enfraquece o sistema imune (Råberg, Grahn, Hasselquist, \& Svensson, 1998), deteriorando a capacidade do organismo responder a qualquer injúria, principalmente a infecções (como a COVID-19). Portanto, um paciente ansioso, estressado e imunodeprimido tem muito mais chance de, caso contraia o coronavírus, desenvolver formas mais graves e até letais da doença.

\section{COVID-19: o Papel da Transformação Digital}

Transformação digital pode ser compreendida como o processo de mudança realizado a partir do uso das chamadas tecnologias sociais, móveis, analíticas e tecnologias de computação em nuvem (Social, Mobile, Analytics and Cloud - SMAC), que afetam significativamente três ou mais dimensões: no nível individual, empresarial e/ou social (Toolboom, 2016). Estas tecnologias são reconhecidas como aquelas capazes de gerar inovações empresariais que afetam a vida social e econômica de uma nação (Fitzgerald, Kruschwitz, Bonnet, \& Welch, 2013). Uso de dispositivos vestíveis inteligentes (smartbands) para coleta e monitoramento dos dados vitais de pacientes, telemedicina, tecnologias de Internet das Coisas (Internet of Things) e sistemas de informação eletrônicos de apoio à decisão são alguns exemplos de tais tecnologias transformadoras.

Transformação digital não é um assunto novo, já que, nos últimos anos, ele tem sido tema central em diversos setores da sociedade, incluindo negócios, economia e indústria, sendo fortemente recomendado por autores da área. Por exemplo, transformação digital e indústria 4.0 foram temas do Fórum Econômico Mundial de Davos em 2016 (World Economic Forum, 2016). Enquanto o conceito de "transformação digital" e sua condução prática é uma preocupação em empresas de sucesso como Google, Amazon, Apple, Netflix, o uso de tecnologias que promovem a transformação digital ainda é feito de maneira tímida na área de saúde pública no Brasil e em muitos países, incluindo aqueles considerados "desenvolvidos".

Como discutido em Tardieu, Daly, Esteban-Lauzán, Hall, e Miller (2020), o cuidado à saúde vem enfrentando cada vez mais o desafio do crescimento e envelhecimento da população. De acordo com esses autores, a disponibilidade futura de assistência médica, a experiência do paciente, a eficácia do tratamento, a capacidade de assistência médica e a eficiência do sistema dependerão do sucesso das plataformas de troca de informações sobre saúde e da alavancagem dos registros eletrônicos de saúde. Nesse sentido, o EPIDOR busca contribuir com a transformação digital ao permitir a coleta integrada de dados de dores crônicas em instituições de saúde do Brasil e análises dos dados obtidos. Tais informações podem ser aplicadas diretamente no controle da dor crônica e também em estratégias de saúde pública e gestão dos recursos ligados aos tratamentos da dor, os quais possuem custos elevados a municípios, estados e federação.

Ricciardi et al. (2019) também fornecem uma visão interessante de como governar a transformação digital dos serviços de saúde. Os autores mencionam que, semelhante a outras inovações e (novas) tecnologias, essas promessas podem ou não se materializar e trazer benefícios potenciais. Como resultado disso, a introdução, a implementação, a utilização e o financiamento de tecnologias digitais de saúde devem ser cuidadosamente avaliados e monitorados. Os governos devem desempenhar um papel mais ativo na otimização adicional do processo de tomada de decisão, tanto no nível central quanto no descentralizado. Além disso, é necessária uma preparação mais ampla do sistema de saúde para poder lidar com a digitalização, seja através de normas e leis regulatórias, bem como da preparação e treinamento dos profissionais de saúde diante das novas tecnologias.

Outra área que tem contribuído amplamente para o processo de transformação digital é o paradigma da Internet das Coisas (Internet of Things - IoT). Nos cenários de IoT, objetos conectados em rede (por exemplo, smartbands ) produzem e pré-processam dados em tempo real. Nesse contexto, surgem diferentes tipos de aplicações para área de saúde, tais como: i) coleta e análise de dados dos sinais vitais de pacientes; ii) monitoramento de idosos e grupos de pacientes com alguma doença específica; iii) sensoriamento de ambientes de difícil acesso e inóspitos (Sundmaeker, Guillemin, Friess, \& Woelfflé, 2010); iv) aplicações relacionadas à telemedicina (Lei $\mathrm{n}^{\circ}$ 13.989, 2012; Rocha et al., 2016); v) desenvolvimento de sistemas inteligentes para assistência e consulta médica remota; vi) ferramentas computacionais de análise de dados médicos para diagnósticos e auxílio a tomadas de decisão; entre outras.

No atual cenário de pandemia da COVID-19, dispositivos móveis e smartbands podem ser de grande utilidade no monitoramento de indivíduos a distância, evitando seus deslocamentos até hospitais, auxiliando profissionais de saúde no diagnóstico antecipado de pessoas com sintomas da COVID-19 e menor contato físico destes profissionais 
com pacientes infectados. Uma pesquisa em andamento com experimentos práticos que corrobora com tal ideia é apresentada em Nascimento et al. (2020), da qual fazem parte os autores do presente artigo. É apresentada uma abordagem de transformação digital no contexto de atenção primária da saúde pública, no qual smartbands são utilizados para monitorar sinais vitais de grupos de diferentes voluntários com avaliação de seus comportamentos por um sistema de recomendação, além de um software de simulação do movimento dos indivíduos monitorados com apresentação de cenários preditivos de contaminação pela COVID-19.

\section{O Ambiente Computacional EPIDOR}

Diante da necessidade de se mapear e compreender melhor a dor crônica no sistema de saúde brasileiro, sistemas de informação eletrônicos são ferramentas indispensáveis para a tomada de decisão, como ferramenta de avaliação e controle pelo gestor, como preconiza o Protocolo Clínico e Diretrizes Terapêuticas de Dor Crônica (Ministério da Saúde, 2012). No contexto atual, sistemas de informação dessa natureza contribuem para o processo de transformação digital na área de saúde ao fornecer informações relevantes mais rapidamente para tomada de decisão.

Denominado EPIDOR, o ambiente computacional apresentado neste artigo é composto por dois componentes de software: i) um sistema web para coleta e gerência de dados sobre dores crônicas dos pacientes atendidos em instituições de saúde (descrito em subseção seguinte); e ii) um aplicativo móvel, disponibilizado na Play Store, direcionado a pacientes com dores crônicas para que estes possam registrar as medicações tomadas e a intensidade (nível) da dor diária (descrito na subseção seguinte). A ideia com a utilização destes dois tipos de software é fornecer um estudo mais abrangente de dor crônica e outro com grupos específicos. A partir de tal estratégia é possível conferir maior objetividade e precisão na aferição e controle do fenômeno da dor.

\section{METODOLOGIA DE PESQUISA}

Como metodologia de pesquisa, deve-se destacar que o EPIDOR foi desenvolvido com base no levantamento de requisitos sobre dor crônica na literatura da área e com especialistas em medicina da dor, os quais são autores no artigo e membros das Sociedades Brasileiras de Neurocirurgia e de Coluna, além de um deles ser coordenador do Centro de Reabilitação do Hospital da rede FHEMIG envolvido neste projeto. Tais especialistas fizeram também a validação dos requisitos do sistema desenvolvido.

Considerando os critérios de classificação de pesquisa: objetivos, finalidade, abordagem do problema e procedimentos técnicos para coleta de dados, de acordo com Marconi e Lakatos (2002) e Gil (2019), a presente pesquisa pode ser classificada como exploratória e descritiva quanto aos objetivos, pesquisa aplicada quanta à sua finalidade, pesquisa híbrida (quantitativa e qualitativa) quanto a abordagem do problema, e como pesquisa bibliográfica e um estudo de caso quanto aos procedimentos usados para coleta de dados.

Quanto aos procedimentos de coleta de dados, será conduzido um estudo de caso em um hospital da rede pública e uma clínica especializada em medicina da dor com uma amostra de pacientes portadores de dor crônica, os quais terão registrados sua intensidade da dor diária ao longo de um período, sua correlação com atividades e horário de medicação. O processo de coleta de dados sobre dor crônica com o EPIDOR será permanente, isto é, uma vez em funcionamento, o sistema será aplicado como uma ferramenta de coleta de informação continuada, com o objetivo de preencher as atuais lacunas existentes na epidemiologia da dor, uma vez que poderá ser utilizado em ambulatórios da rede pública ou privada e serviços que atendem pacientes com dor crônica.

No que se refere à autorização para coleta de dados dos pacientes, serão registrados no EPIDOR todos os pacientes que procurarem o serviço de dor e, uma vez que os dados coletados e armazenados durante a consulta médica têm caráter de confidencialidade e são tratados como parte dos prontuários médicos, essa medida por natureza já implica em fonte sigilosa de conhecimento médico, desde que respeitada a confidencialidade e privacidade do paciente conforme as normas da Resolução CFM n. 1.639/02.

\section{Sistema Web EPIDOR}

O componente sistema web do EPIDOR é responsável pela coleta de dados de dores crônicas de todos pacientes atendidos pela instituição de saúde que adotará o sistema, bem como provê análise dos dados coletados a partir da geração de dashboards. O acesso ao sistema web do EPIDOR pode ser feito a partir de qualquer browser por computadores desktops, tablets ou smartphones, eliminando custos desnecessários para a instituição de saúde na aquisição de computadores com configurações específicas ou servidores.

Apenas o profissional de saúde que acompanha o paciente tem login e senha de acesso ao sistema web, as quais são criptografadas utilizando algoritmo de hash. O profissional de saúde cadastra-se no sistema e recebe um token para confirmar sua identidade para segurança.

Após o login no sistema, o profissional de saúde pode realizar o preenchimento do formulário da dor, que corresponde à atividade principal na abordagem de pesquisa adotada. Como estratégia de coleta de dados nessa atividade, foi utilizado o formulário da dor validado em língua portuguesa - conhecido como Inventário Breve 
de Dor (do inglês Brief Pain Inventory - BPI) - instrumento multidimensional, que faz uso de uma escala de 0 a 10 para classificar os seguintes itens: intensidade, interferência da dor na habilidade para caminhar, atividades diárias do paciente, no trabalho, atividades sociais, humor e sono (Pimenta, 1994).

O preenchimento do Inventário Breve de Dor no sistema se dá em três etapas: na etapa 1 informa-se a localização da dor em regiões do corpo humano (vide Figura 1); na etapa 2 deve-se informar a classificação da dor (vide Figura 2) e na etapa 3 o grau de interferência da dor nas atividades da vida diária, como humor, relacionamento interpessoal, habilidade de caminhar, sono, trabalho e avaliação da vida (vide Figura 3).

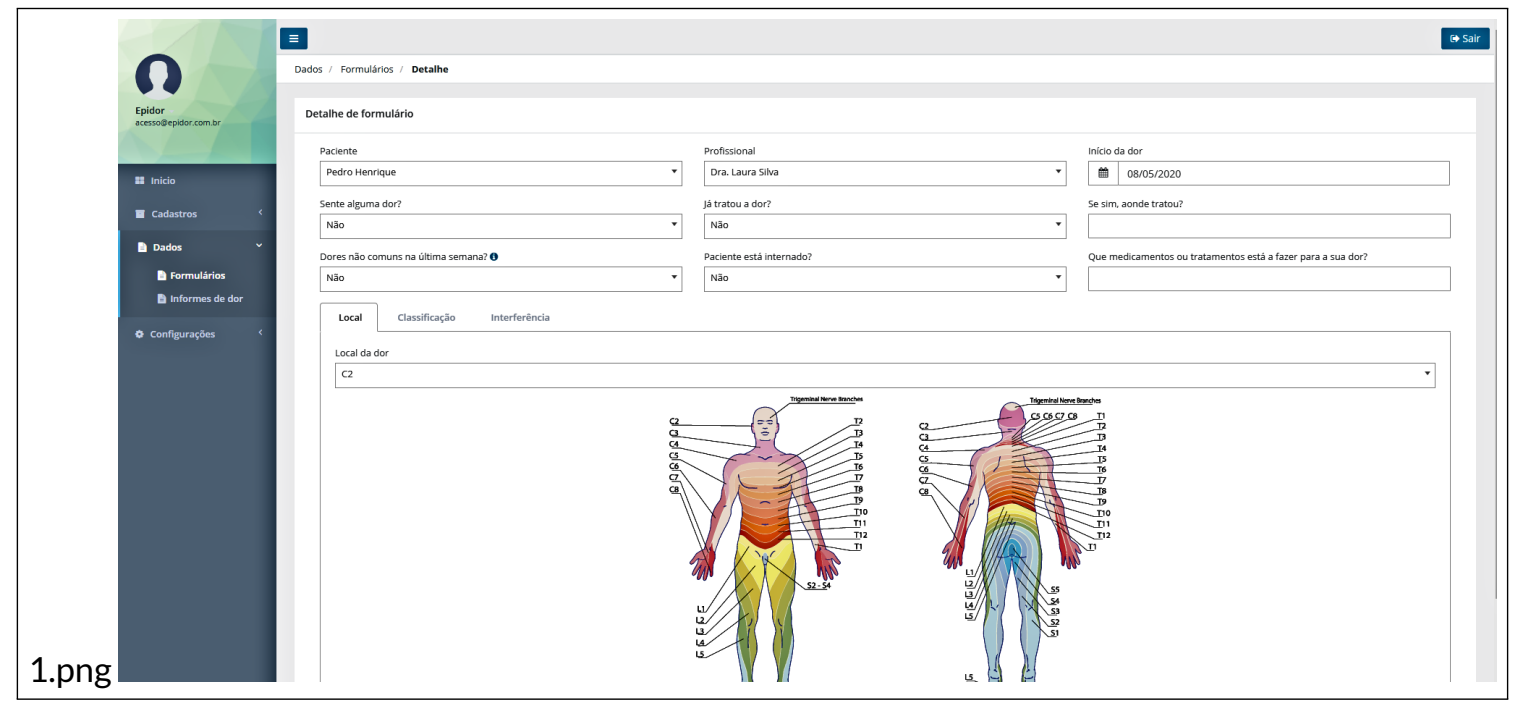

Figura 1. Parte do formulário da dor para registro de sua localização no corpo

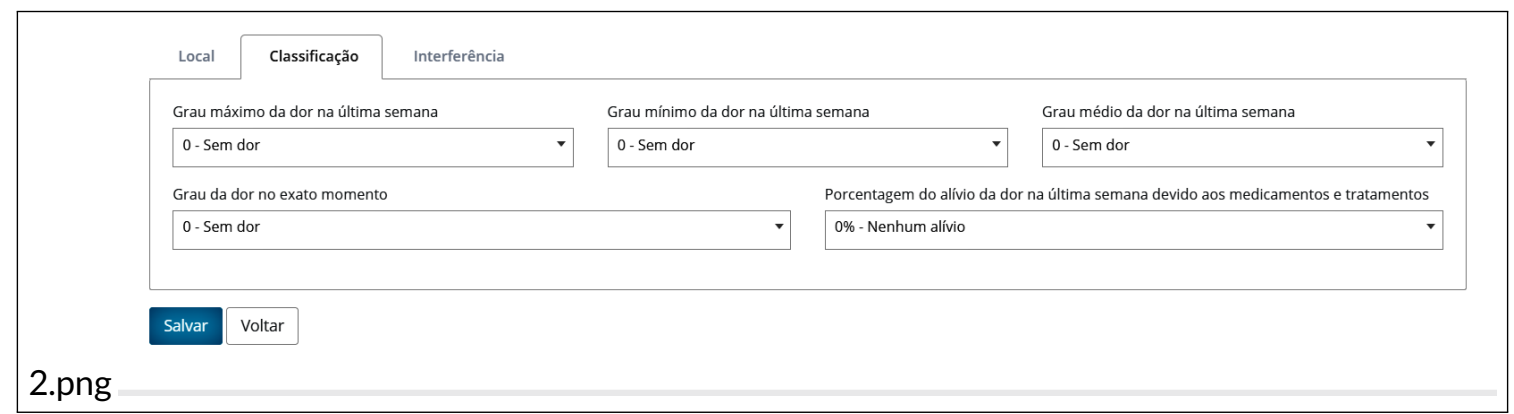

Figura 2. Parte do formulário da dor para registro de sua classificação

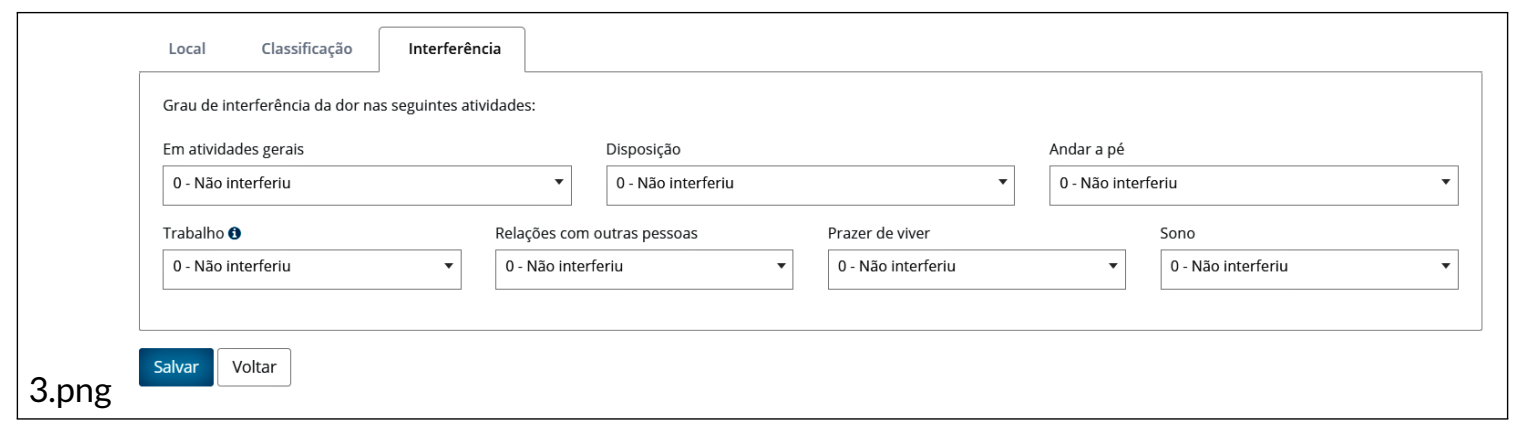

Figura 3. Parte do formulário da dor para registro de seu grau de interferência

Por fim, é importante destacar as tecnologias usadas no desenvolvimento do sistema web do EPIDOR. Foram utilizados o IDE Visual Studio 2019 Community como ambiente de desenvolvimento, o template INSPINIA Responsive Admin Theme para programação front-end nas linguagens HTML5, CSS3 e JavaScript, e o banco de dados SQL Server. Os dados coletados são armazenados nesse banco de dados localizado em um serviço em nuvem de um servidor de hospedagem, o qual oferece mecanismos de proteção de acesso aos dados.

\section{Aplicativo móvel do EPIDOR}


O outro estudo de dor crônica proposto nesta pesquisa é realizado através do aplicativo móvel do EPIDOR, desenvolvido para coletar dados diariamente de grupos de pacientes, por meio de uma interface simples e intuitiva aos seus usuários para registro das medicações tomadas e a intensidade da dor. A partir dessa medida é possível acompanhar a evolução clínica de cada paciente no tratamento da dor e prover cuidados diferenciados para cada situação específica. Diferente do sistema web, o acesso ao aplicativo móvel é feito pelo próprio paciente com orientação do profissional de saúde que lhe acompanha e realiza junto com o paciente o login e primeiro acesso. Além disso, os pacientes receberão treinamento para utilização do aplicativo móvel e sempre terão meios de acesso fácil para contatar seu médico assistente em caso de dúvidas com o sistema. No caso dos idosos, caso eles tenham um cuidador, este pode ser treinado para uso do aplicativo. O aplicativo móvel, denominado Diário Digital da Dor, permite acompanhar o nível da dor do paciente em momentos predeterminados do dia e avisar sobre o horário das próximas doses da medicação usada. O cadastro e primeiro login na aplicação devem ser realizados ao lado de um profissional de saúde, responsável pelo atendimento desse paciente. As interações seguintes com o aplicativo já podem ser feitas pelo próprio paciente de qualquer lugar. A Figura 4, a seguir, ilustra a interface do aplicativo Diário Digital da Dor e também o menu principal com acesso às funcionalidades de aviso de medicamentos e aviso do nível da dor. No registro da medicação e do nível da dor são armazenadas também data e hora da coleta dos dados, as quais são essenciais para o estudo, a fim de traçar uma rotina mais saudável para o paciente, bem como verificar a eficácia da medicação.

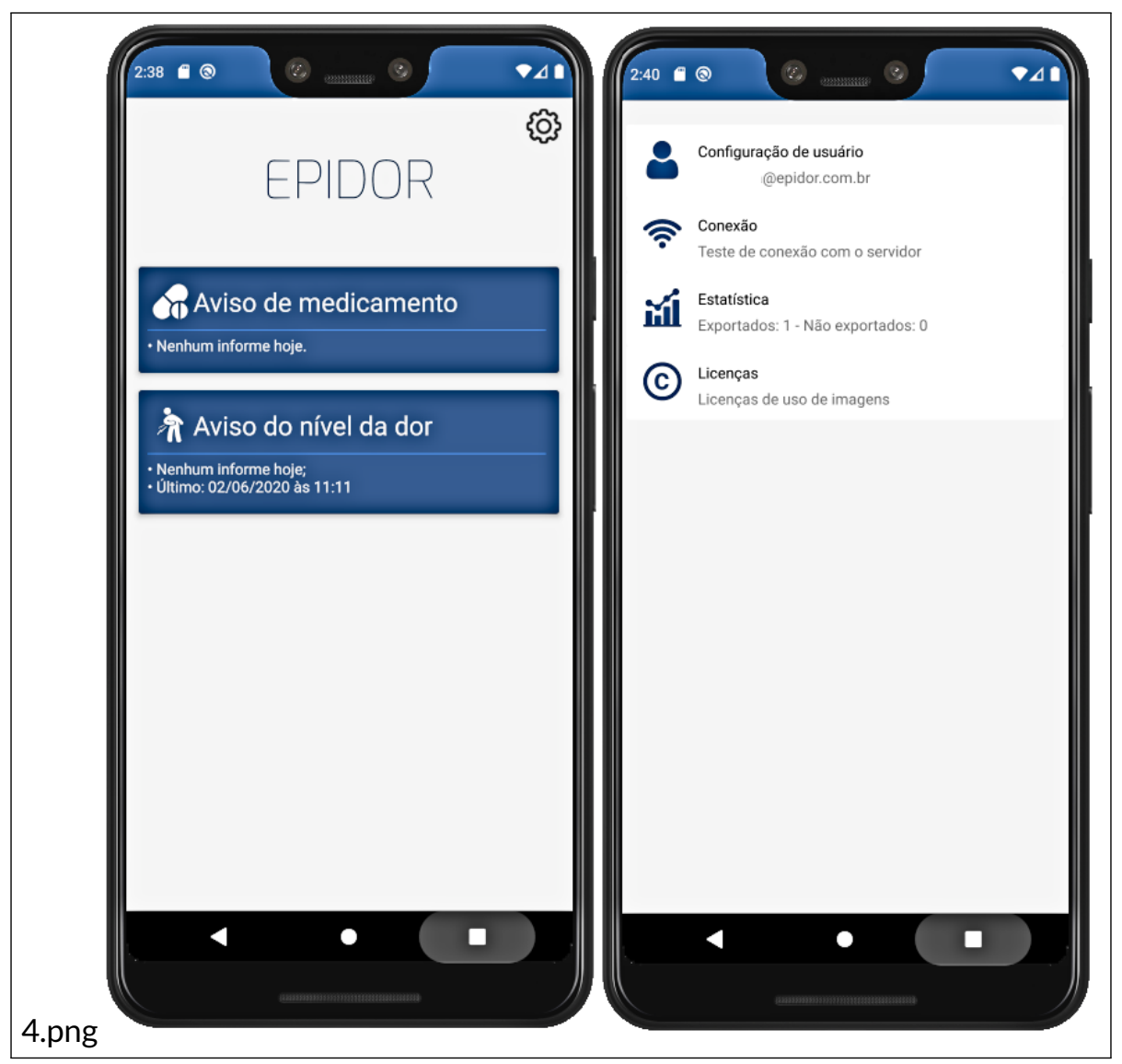

Figura 4. Interface do Menu Principal do Aplicativo

Na função de registro do nível da dor (vide Figura 5), foi utilizada uma escala de dor similar à Escala Visual Analógica (EVA), considerado um tipo de medição da dor simples e fidedigno à realidade diária do paciente, que permite avaliar fenômenos subjetivos associados à dor, percepção da sensibilidade e avaliação da dor de modo geral (Vles et al., 2008). Foram utilizadas cores diferentes para cada nível de intensidade (0 - Sem dor; 1 - Suave; 2 - Leve; 3 - Desconfortável; 4 - Moderada Leve; 5 - Moderada; 6 - Moderada Forte; 7 - Forte; 8 Severa; 9 - Extrema; 10 - Indescritível), além de botões maiores, com o propósito de facilitar o registro da dor por parte do usuário. Essa é uma medida essencial quando o público-alvo do aplicativo pode incluir usuários com alguma dificuldade no uso de recursos eletrônicos, tais como pacientes com dor crônica. Quanto às tecnologias, o aplicativo móvel foi desenvolvido em Java, utilizando o ambiente de desenvolvimento Android Studio e pode ser executado em qualquer dispositivo que utiliza o Android na versão Jelly Bean (4.1.x) ou superior.

Figura 5. Interface de Registro do Nível da Dor 


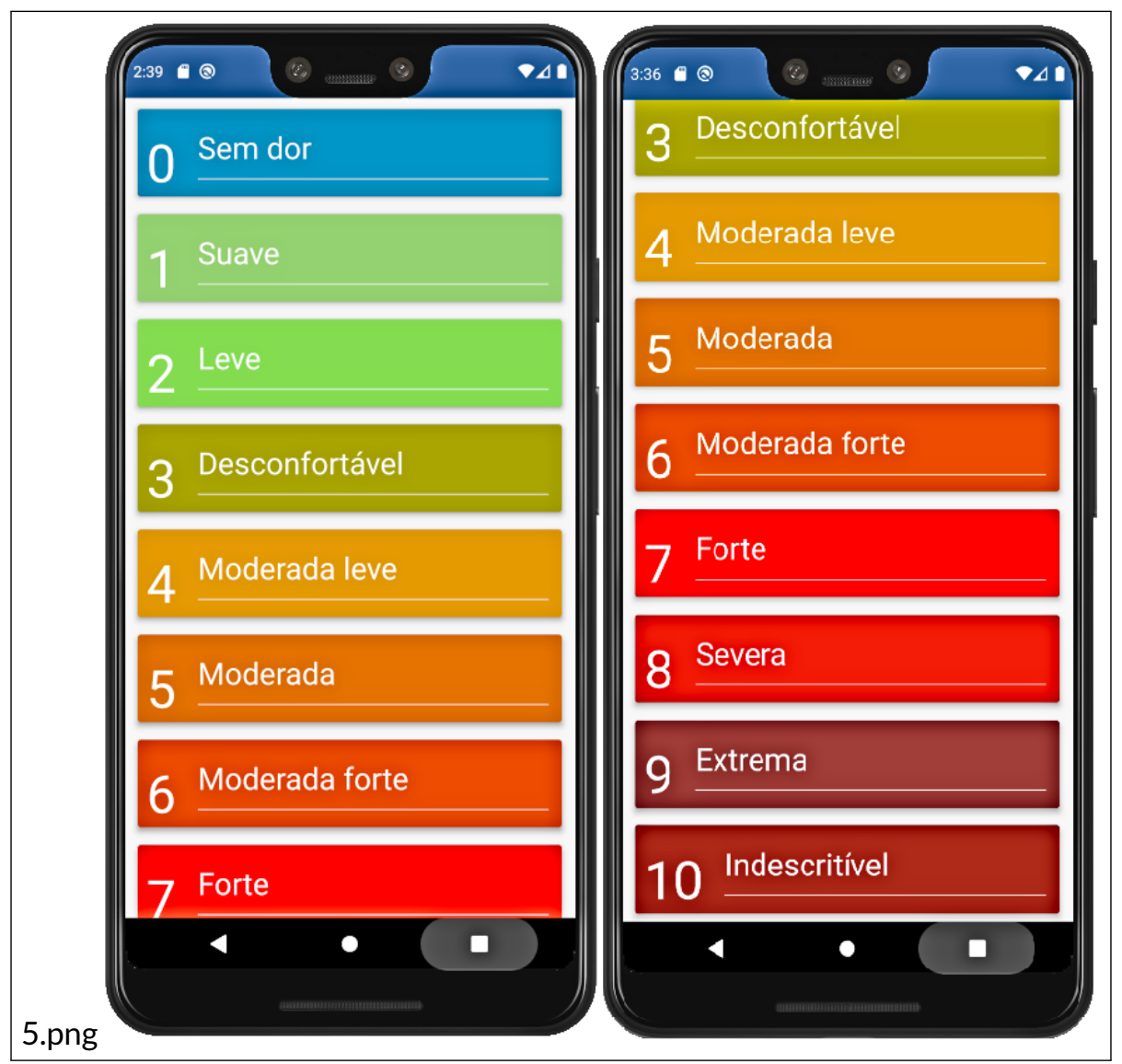

Figura 5. Interface de Registro do Nível da Dor

A partir dos registros feitos no aplicativo do Diário Digital da Dor, é possível acompanhar a evolução do tratamento da dor e fornecer aos médicos condições de monitorar o cotidiano do paciente quanto às ações que influenciam no grau ou natureza da dor e também contribuindo para ajustes medicamentosos e tratamento específicos.

\section{DISCUSSÃO E RESULTADOS ESPERADOS}

Estudos epidemiológicos que avaliam a dor na forma de questionários práticos e de fácil aplicabilidade são importantes para informar aos gestores de saúde sobre os impactos na população e também para o controle da dor crônica. Porém, tais estudos ainda não foram devidamente conduzidos, em virtude de diversas barreiras metodológicas na coleta dos dados da dor crônica como um único fenômeno e também de elevados custos para se estruturar uma base de dados padronizada. Diante disso, uma solução apropriada para mapear e compreender melhor a dor crônica no sistema de saúde brasileiro é o uso de tecnologias e sistemas de informação para tomada de decisão na área, tal como o EPIDOR apresentado no presente artigo.

É importante destacar que o EPIDOR faz parte de uma pesquisa em andamento e, por esse motivo, não foram apresentados dados coletados sobre dor crônica neste artigo, os quais poderiam apoiar estudos epidemiológicos na área. Atualmente, o EPIDOR encontra-se ainda em fase de implantação em um hospital de referência para atendimento de municípios da região da Zona Mata mineira, localizado na cidade de Juiz de Fora, e também em uma clínica especializada em medicina da dor deste mesmo município. No hospital citado, será conduzido o estudo mais geral sobre dores crônicas desta pesquisa, que abrange a coleta e gerenciamento dos dados de todos pacientes com dor crônica atendidos no hospital, através do registro no formulário eletrônico Breve Inventário da Dor do sistema web do EPIDOR. Na clínica especializada, conduz-se o estudo mais específico de dor crônica com um grupo de pacientes selecionado para uso do aplicativo móvel do EPIDOR, que permite acompanhar a evolução clínica destes pacientes, o controle de qualidade do atendimento e do tratamento realizado. Como citado anteriormente, a partir da estratégia desses dois estudos é possível conferir maior objetividade e precisão na aferição do fenômeno da dor.

A partir da conclusão de implantação do sistema e dos resultados obtidos no hospital e clínica citados, o projeto do ambiente computacional EPIDOR poderá ser expandido para outras instituições de saúde regionais e nacionais, tornando-se um instrumento valioso no suporte às estratégias de saúde relacionadas à gestão da dor crônica. Em outras palavras, a implantação e utilização do EPIDOR na cidade de Juiz de Fora - quarto município mineiro mais populoso - servirá de indicação de teste de conceito do projeto para ações maiores em nível nacional. 
Como contribuição do EPIDOR no contexto de transformação digital para epidemiologia da dor, espera-se que o sistema, quando em pleno funcionamento na rede pública de saúde possa gerar informações mais rápidas e precisas sobre o estado de saúde dos pacientes acompanhados, possibilitando detecção precoce da qualidade da dor e situações de emergência; economia de custos operacionais na coleta e gestão de dados sobre dores crônicas; e provimento de suporte às tomadas de decisão gerencial sobre estratégias e políticas públicas de saúde no enfrentamento da pandemia da COVID-19 relacionados à dor crônica.

A relação entre dor crônica e COVID-19 não se dá por conta da dor crônica em si, já que esta é crônica. Entretanto, um paciente portador de dor crônica que sofre uma agudização da mesma, pode necessitar de intervenção médica mais urgente, e, em virtude da pandemia, esses pacientes podem relutar em procurar socorro médico por medo de uma possível contaminação. Nesses casos, assim que o paciente sinalizar pelo aplicativo um agravamento da intensidade de sua dor crônica, o médico será prontamente comunicado pelo sistema e pode entrar em contato com o paciente a fim de investigar melhor a possível causa do agravamento da dor. Dessa maneira, o paciente, caso tenha uma agudização da dor por uma possível emergência médica (por exemplo, infarto do miocárdio, fratura, cólica nefrética etc.), seria orientado pelo médico a procurar socorro a tempo. É dentro desse contexto que se dá a intersecção entre o sistema EPIDOR e a situação oriunda da pandemia da COVID-19.

Acrescenta-se ainda a motivação dos pacientes para fazer o acompanhamento através do EPIDOR. A escolha e a dosagem dos medicamentos podem variar - e, normalmente, varia - em função das características que a dor venha assumir. Um acompanhamento mais estrito, próximo e célere, como a proposta do EPIDOR, possibilita um ajuste mais fino da escolha dos medicamentos bem como de sua posologia. Os pacientes, sabendo dessas vantagens, podem sentir-se mais motivados a aderir ao tratamento, já que estarão sendo monitorados de uma maneira bem mais eficiente.

\section{CONSIDERAÇÕES FINAIS}

O presente artigo apresentou um ambiente computacional para coleta, acompanhamento e gestão de dados de pacientes com dores crônicas, denominado EPIDOR (acrônimo de Epidemiologia da Dor), cujo objetivo principal é contribuir com estudos epidemiológicos mais completos e detalhados sobre dor crônica no Brasil, os quais carecem de tal necessidade. O sistema é composto por dois componentes de software (um sistema web e um aplicativo móvel), que possibilitam a coleta de dados de dores crônicas, registro eletrônico da intensidade da dor ao longo de um período, sua correlação com atividades e horário de medicação, além da geração de dashboards dos dados obtidos a fim de prover análise de dados para tomada de decisão gerencial.

Como pesquisa em andamento, o EPIDOR encontra-se ainda em fase de implantação em um hospital regional da cidade de Juiz de Fora e em uma clínica especializada em medicina da dor deste mesmo município. A partir da conclusão de implantação do sistema e dos resultados obtidos no hospital e clínica citados, o projeto do ambiente computacional EPIDOR poderá ser expandido para outras instituições de saúde regionais e nacionais, tornando-se um instrumento valioso no suporte às estratégias de saúde relacionadas à gestão da dor crônica.

Outro propósito com a utilização do EPIDOR no âmbito de instituições de saúde que prestam serviços no tratamento da dor crônica é trazer contribuições para a transformação digital na área, medida imprescindível no contexto atual. O sistema EPIDOR, em sua concepção de produto de software, envolve as chamadas tecnologias sociais, móveis, analíticas e de computação em nuvem que caracterizam processos de transformação digital. Tecnologias móveis do EPIDOR foram empregadas no desenvolvimento do aplicativo móvel; a computação em nuvem está presente na configuração do ambiente computacional do sistema web, que utiliza servidores de aplicação, hospedagem e banco de dados em nuvem; tecnologias analíticas fazem-se presentes no EPIDOR na geração de dashboards dos dados coletados e processamento dos mesmos através de técnicas de análise de dados (data analytics); e por fim, o EPIDOR caracteriza-se como tecnologia social no que tange ao seu aspecto de promover melhoria na qualidade de vida da população com dor crônica ao fornecer recursos para acompanhamento do controle e tratamento da dor dos pacientes pelos profissionais de saúde, possibilitando intervenções especializadas em cada caso específico.

O tema transformação digital na saúde ganha maior relevância no contexto atual de pandemia do coronavírus, porque estamos enfrentando não apenas uma crise nos serviços de saúde prestados à população, mas também a falta de informações corretas, precisas e relevantes sobre a COVID-19, que englobam números reais da doença, de mortalidades, leitos de enfermaria e UTIs para atendimento à doença, índices de transmissão do vírus, índices de mobilidade urbana, entre outros dados imprecisos em muitas fontes de informação. Diante desse cenário, a adoção de tecnologias com capacidade de transformação e sistemas eletrônicos eficientes podem trazer grandes contribuições aos gestores públicos e a população como um todo.

No atual cenário de pandemia da COVID-19, é importante destacar também que as tecnologias com capacidade de transformação devem vir acompanhadas de estratégias de enfrentamento e intervenções que incluem as dimensões sociais (por exemplo, assistência social e apoio familiar) e psicológicas (por exemplo, acompanhamento 
através da psicoterapia on-line, psiquiatria on-line e teleconsulta) (Pereira et al., 2020). Logo, em tempos de crise é fundamental criar manejos para o fortalecimento da saúde mental do indivíduo (Ho, Chee, \& Ho, 2020).

Um bom exemplo que o Brasil pode seguir vem de experiências realizadas na China com o uso de tecnologias de assistência médica e apoio psicológico. Por exemplo, o sistema de serviços de saúde mental chinês foi bastante aprimorado após vários desastres importantes, especialmente o terremoto de Wenchuan. Estratégias similares têm sido adotadas no país para tratar problemas psicológicos urgentes das pessoas envolvidas na pandemia da COVID-19. Em um modelo que utiliza tecnologias da Internet, o West China Hospital integra médicos, psiquiatras, psicólogos e assistentes sociais em plataformas da Internet para realizar intervenção psicológica para pacientes, suas famílias e equipe médica. A ideia central é unir a tecnologia da Internet ao processo de intervenção, bem como combinar intervenções precoces com reabilitação posterior (Zhang, Wu, Zhao, \& Zhang, 2020). Tais medidas aliadas a processos de transformação digital mostram-se apropriadas para melhor enfrentamento dos desafios e problemas impostos pela atual pandemia da COVID-19.

\section{AGRADECIMENTO}

Agradecemos o apoio financeiro concedido por: Programa Pesquisa para o SUS (gestão compartilhada em saúde - PPSUS), FAPEMIG (processo APQ-04202-17), DECIT/SCTIE/MS, CNPq e SES/MG. 


\section{REFERÊNCIAS}

Andrade, R. M. Q. (2014). Dor crônica na atenção primária: um problema de saúde pública (Monografia de Especialização em Atenção Básica em Saúde da Família, Universidade Federal de Minas Gerais. Faculdade de Medicina. Núcleo de Educação em Saúde Coletiva, Juiz de Fora, MG, Brasil). Recuperado de https://www.nescon.medicina.ufmg.br/ biblioteca/imagem/4601.pdf

Carvalho, R. C. d., Maglioni, C. B., Machado, G. B., Araújo, J. E. d., Silva, J. R. T. d., \& Silva, M. L. d. (2018). Prevalence and characteristics of chronic pain in brazil: a national internet-based survey study. BrJP, 1(4), 331-338.

Dellaroza, M. S. G., Pimenta, C. A. d. M., \& Matsuo, T. (2007). Prevalência e caracterização da dor crônica em idosos não institucionalizados. Cadernos de saúde pública, 23(5), $1151-1160$.

Fishbain, D. A., Lewis, J. E., Cole, B., Cutler, R. B., Rosomoff, H. L., \& Rosomoff, R. S. (2007). Variables associated with current smoking status in chronic pain patients. Pain Medicine, 8(4), 301-311.

Fitzgerald, M., Kruschwitz, N., Bonnet, D., \& Welch, M. (2013). Embracing digital technology: A new strategic imperative. MIT Sloan Management Review.

Gil, A. C. (2019). Como elaborar projetos de pesquisa (6a. ed.). São Paulo: Atlas.

Haddad, J. J., Saadé, N. E., \& Safieh-Garabedian, B. (2002). Cytokines and neuro-immune-endocrine interactions: a role for the hypothalamic-pituitary-adrenal revolving axis. Journal of neuroimmunology, 133(1-2), 1-19.

Ho, C. S., Chee, C. Y., \& Ho, R. C. (2020). Mental health strategies to combat the psychological impact of covid-19 beyond paranoia and panic. Ann Acad Med Singapore, 49(3), 155-160. Recuperado de https://pubmed.ncbi.nlm.nih.gov/ 32200399

Hossain, M. M., Sultana, A., \& Purohit, N. (2020). Mental health outcomes of quarantine and isolation for infection prevention: A systematic umbrella review of the global evidence. PsyArXiv Preprints, 1-27. doi: https://doi.org/10.31234/OSF.IO/DZ5V2

Kreling, M. C. G. D., Cruz, D. A. L. M. d., \& Pimenta, C. A. M. (2006). Prevalência de dor crônica em adultos. Revista Brasileira de Enfermagem, 59(4), 509-513.

Lei $\mathrm{n}^{\mathrm{o}}$ 13.989. (2012). Dispõe sobre o uso da telemedicina durante a crise causada pelo coronavírus (SARS-CoV-2). Recuperado de http://www.in.gov.br/web/dou/-/lei-n-13 .989-de-15-de-abril-de-2020-252726328

Lima, C. K. T., Carvalho, P. M. M., Lima, I. A. S., Nunes, J. V. A., Saraiva, J. S., Souza, R. I., ... Neto, M. L. R. (2020). The emotional impact of coronavirus 2019-ncov (new coronavirus disease). Psychiatry research, 287, 112915. doi: https://doi.org/10.1016/j.psychres.2020.112915

Marconi, M. A., \& Lakatos, E. M. (2002). Técnicas de pesquisa: planejamento e execução de pesquisas, amostragens e técnicas de pesquisas, elaboração, análise e interpretação de dados (5a. ed.). São Paulo: Atlas.

Minghelli, B., Soares, A., Guerreiro, A., Ribeiro, A., Cabrita, C., Vitoria, C., ... others (2020). Physiotherapy services in the face of a pandemic. Revista da Associação Médica Brasileira, 66 (4), 491-497. Recuperado de https://dx.doi .org/10.1590/1806-9282.66.4.491

Ministério da Saúde. (2012). Portaria SAS/MS no 1083, de 02 de outubro de 2012. Aprova o Protocolo Clínico e Diretrizes Terapêuticas Dor Crônica. Retificada em 27 de novembro de 2015. Recuperado de http://bvsms.saude.gov .br/bvs/saudelegis/sas/2012/prt1083 $02 \quad 10$ 2012.html

Nascimento, M. G., Iorio, G., Thomé, T. G., Medeiros, A. A., Mendonça, F. M., Campos, F. A., ... Dantas, M. A. (2020). Covid-19: A digital transformation approach to a public primary healthcare environment. In Proceedings ieee symposium on computers and communications (iscc).

Organização Pan-Americana da Saúde [OPS/OMS]. Unidade de Saúde Mental e Uso de Substâncias. (2016). Protección de la salud mental y atención psicosocial en situaciones de epidemias. Recuperado de https://www.paho.org/ disasters $/$ index.php?option $=$ com_docman\&view $=$

download\&category slug $=$ informes-tecnicos\&alias $=2539$ -proteccion-salud-mental-atencion-psicosocial-situaciones -epidemias-2016-539\&Itemid=1179\&lang=en

Pereira, M. D., Oliveira, L. C., Costa, C. F. T., Bezerra, C. M. O., Pereira, M. D., Santos, C. K. A., \& Dantas, E. H. M. (2020). The covid-19 pandemic, social isolation, consequences on mental health and coping strategies: an integrative review. Research, Society and Development, 9(7), 1-35. doi: http://dx.doi.org/10.33448/rsd-v9i7.4548

Pimenta, C. A. M. (1994). Escalas de avaliação de dor. dor conceitos gerais. São Paulo: Limay.

Queiroz, L. P., Barea, L. M., \& Blank, N. (2006). An epidemiological study of headache in florianopolis, brazil. Cephalalgia, 26(2), 122-127.

Råberg, L., Grahn, M., Hasselquist, D., \& Svensson, E. (1998). On the adaptive significance of stress-induced immunosuppression. Proceedings of the Royal Society of London. Series B: Biological Sciences, 265(1406), 1637-1641.

Rede Diário de Comunicação. (2020). Medo de covid-19 faz pacientes de fibromialgia não buscarem ajuda. Recuperado de https://d24am.com/saude/medo-de-covid-19-faz -pacientes-de-fibromialgia-nao-buscarem-ajuda/

Ricciardi, W., Barros, P. P., Bourek, A., Brouwer, W., Kelsey, T., \& Lehtonen, L. (2019). How to govern the digital transformation of health services. European journal of public health, 29(Supplement_3), 7-12.

Rocha, T. A. H., Fachini, L. A., Thumé, E., Silva, N. C. d., Barbosa, A. C. Q., Carmo, M. d., \& Rodrigues, J. M. (2016). Saúde móvel: novas perspectivas para a oferta de serviços em saúde. Epidemiologia e Serviços de Saúde, 25(1), 159-170.

Sá, K., Baptista, A. F., Matos, M. A., \& Lessa, I. (2009). Prevalência de dor crônica e fatores associados na população de salvador, bahia. Revista de Saúde Pública, 43(4), 622630 .

Sundmaeker, H., Guillemin, P., Friess, P., \& Woelfflé, S. (2010). Vision and challenges for realising the internet of things. Cluster of European Research Projects on the Internet of Things, European Commision, 3(3), 34-36. Recuperado de https://docbox.etsi.org/zArchive/TISPAN/Open/ IoT/CERP-IOT_Clusterbook_2009.pdf

Tardieu, H., Daly, D., Esteban-Lauzán, J., Hall, J., \& Miller, G. (2020). Case study 2: The digital transformation of health care. In Deliberately digital. future of business and finance. springe, cham. doi: https://doi.org/10.1007/978-3030-37955-1_23

Teixeira, M. J., Teixeira, W. G. J., Santos, F. P. S., Andrade, D. C. A., Bezerra, S. L., Figueiró, J. B., \& Okada, M. (2001). Epidemiologia clínica da dor músculo-esquelética. Revista de Medicina, 80, 1-21. 
Toolboom, I. (2016). The impact of digital transformation (Master in System Engineering, Policy analysis and Management). Faculty of Technology, Policy and Management, Delf.

Vachon-Presseau, E., Roy, M., Martel, M.-O., Caron, E., Marin, M.-F., Chen, J., ... others (2013). The stress model of chronic pain: evidence from basal cortisol and hippocampal structure and function in humans. Brain, $136(3), 815-827$.

Vasconcelos, F. H., \& Araújo, G. C. (2018). Prevalence of chronic pain in brazil: a descriptive study. BrJP, 1(2), 176-179.

Vles, G. F., de Louw, A. J., Speth, L. A., van Rhijn, L. W., Janssen-Potten, Y. J., Hendriksen, J. G., \& Vles, J. S. (2008). Visual analogue scale to score the effects of botulinum toxin a treatment in children with cerebral palsy in daily clinical practice. European Journal of Paediatric Neurology, 12(3), 231-238.

Williams, B. (2020). Mental health concerns arise amid covid-19 epidemic. Psychiatry Advisor. Recuperado de https://www.psychiatryadvisor.com/home/topics/ general-psychiatry/mental-health-concerns-arise-amid -covid-19-epidemic/

World Economic Forum. (2016). World economic forum annual meeting.

Yang, Y., Li, W., Zhang, Q., Zhang, L., Cheung, T., \& Xiang, Y.-T. (2020). Mental health services for older adults in china during the covid-19 outbreak. The Lancet Psychiatry, 7(4). doi: https://doi.org/10.1016/S2215-0366(20)30079-1

Zhang, J., Wu, W., Zhao, X., \& Zhang, W. (2020). Recommended psychological crisis intervention response to the 2019 novel coronavirus pneumonia outbreak in china: a model of west china hospital. Precision Clinical Medicine, $3(1), 3-8$.

Mendonça, F. M., Junior, C. A. M., Oliveira, J. F. S., Thomé, T. G., \& Filgueiras, M. Q. (2020). EPIDOR: uma abordagem computacional baseada em sistema web e aplicativo móvel para dores crônicas no atual contexto de pandemia do Coronavírus. AtoZ: novas práticas em informação e conhecimento, 9(2), 117 128. Recuperado de: http://dx.doi.org/10.5380/atoz .v9i2.74673 